\title{
BEAM DYNAMICS STUDIES FOR THE REACCELERATION OF LOW ENERGY RIBS AT THE NSCL*
}

\author{
X. $\mathrm{Wu}^{\dagger}$, G. Bollen, M. Doleans, T. L. Grimm, D. Lawton, F. Marti, S. Schwarz, R. C. York \\ and Q. Zhao \\ National Superconducting Cyclotron Laboratory, Michigan State University, E. Lansing, MI 48824, \\ U.S.A.
}

\section{Abstract}

Rare Isotope Beams (RIBs) are created at the National Superconducting Cyclotron Laboratory (NSCL) by the in-flight particle fragmentation method. A novel system that stops the RIBs in helium gas and reaccelerates them is proposed to provide opportunities for an experimental program ranging from low energy Coulomb excitation to transfer reaction studies of astrophysical reactions. The beam from the gas stopper [1] will first be brought into a Electron Beam Ion Trap (EBIT) charge breeder [2] on a high voltage platform to increase its charge state, and then accelerated up to about $3 \mathrm{MeV} / \mathrm{u}$ by a system consisting of an external multi-harmonic buncher and a radio frequency quadrupole (RFQ) followed by a superconducting linac. The superconducting linac will use quarter-wave resonators with optimum acceleration for particle velocities as a fraction of the speed of light $\left(\beta_{\text {opt }}\right)$ of 0.041 and 0.085 for acceleration and superconducting solenoid magnets for transverse focusing. The accelerator system design and the end-to-end beam dynamics simulations are presented.

\section{INTRODUCTION}

To meet the need for the nuclear astrophysics experimental program, the NSCL reaccelerator is required to accelerate RIBs from the EBIT with charge-to-mass ratios $(\mathrm{Q} / \mathrm{A})$ ranging from 0.2 to 0.4 and to achieve a final energy from 0.3 to $3 \mathrm{MeV} / \mathrm{u}$. The possibility to upgrade the output energy to $12 \mathrm{MeV} / \mathrm{u}$ is also considered. The requirements of the bunch width and the energy spread for the RIBs on target are $1 \mathrm{~ns}$ and $1 \mathrm{keV} / \mathrm{u}$, corresponding to a longitudinal emittance of $\sim 0.25 \pi \mathrm{keV} / \mathrm{u}$-ns. The beam spot on target is required to be $\sim 1 \mathrm{~mm}$. The EBIT will have an extraction voltage ranging from $30 \mathrm{kV}$ for RIBs with $\mathrm{Q} / \mathrm{A}=0.4$ to $60 \mathrm{kV}$ for RIBs with $\mathrm{Q} / \mathrm{A}=0.2$, resulting in a fixed RFQ input energy of $12 \mathrm{keV} / \mathrm{u}$. For the simulations, the normalized transverse beam emittance from the EBIT was assumed to be $0.6 \pi \mathrm{mm}$-mrad with a maximum energy spread of $\pm 0.2 \%$, based on experience with operating EBIS at REX-ISODLE [3,4]. It was observed in the beam simulations that the longitudinal emittance after the RFQ is mainly determined by the initial energy spread of the beam from the EBIT.

The proposed accelerator system consists of four segments: a Low Energy Beam Transport system (LEBT) to transport, bunch and match the RIBs from the EBIT to

\footnotetext{
*Work supported by Michigan State University and NSF

\#xwu@nscl.msu.edu
}

the RFQ, an RFQ for initial beam acceleration and focusing, a superconducting linac system for RIBs acceleration to the desired energy for nuclear astrophysics experimental program, and a High Energy Beam Transport system (HEBT) to deliver the RIBs to the experimental area with the required beam conditions. The entire accelerator system and the experimental area will be located on a balcony in the NSCL high bay area. Figure 1 shows the layout of the proposed accelerator system.

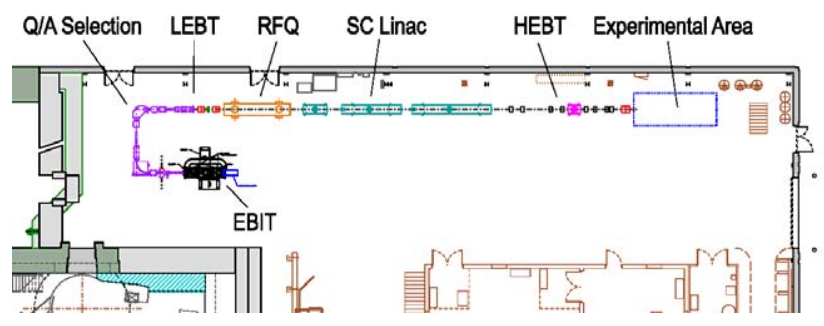

Figure 1: The layout of the proposed accelerator system.

End-to-end beam simulations of the NSCL reaccelerator using various computer codes were performed to evaluate the performance of the proposed accelerator system and determine the hardware specifications. Beam simulations through the LEBT and RFQ were investigated using RIAPMTQ [5]. IMPACT [5] and a code developed at the NSCL [6] were used for beam dynamics studies in the superconducting linac section and HEBT. DIMAD [7] and TRACE3D [8] were used to study transverse focusing, beam matching, and the effect of misalignment and correction scheme in the superconducting linac section.

The reaccelerator design benefits from past $R \& D$ efforts at the NSCL for a heavy ion driver linac [9]. For example, the front-end design was derived from the one for the driver linac. Also, the superconducting linac uses the same $80.5 \mathrm{MHz} \lambda / 4$ cavities as the driver linac. The structure with $\left(\beta_{\mathrm{opt}}\right)$ of 0.085 has already been prototyped and shown to exceed the design requirements [10]. The structure with $\left(\beta_{\text {opt }}\right)$ of 0.041 is being prototyped currently at the NSCL. Finally, the beam simulation tools developed for driver linac were used extensively for the beam dynamics studies.

\section{LEBT}

The LEBT uses four electrostatic quadrupoles and two solenoid magnets to provide transverse focusing. To achieve a small longitudinal emittance, an external MultiHarmonic Buncher (MHB) is used prior to the RFQ. The 
location and the voltages of the different harmonics of the MHB were optimized to match the beam longitudinally into the RFQ acceptance.

The end-to-end beam simulations begin with the RIBs from the EBIT after the Q/A selection with an energy of $12 \mathrm{keV} / \mathrm{u}$ and $\mathrm{Q} / \mathrm{A}$ of 0.25 . The simulations used a beam with normalized transverse emittance of $0.6 \pi$ $\mathrm{mm}$-mrad and an energy spread of $\pm 0.2 \%$ as mentioned above. Figure 2 shows the initial phase space of the RIBs at the entrance of the LEBT.
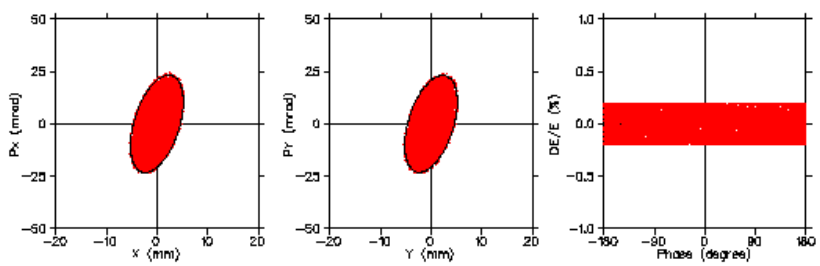

Figure 2: Horizontal (left), vertical (middle), and longitudinal (right) phase space at the entrance of the LEBT. The black ellipses represent the $90 \%$ emittance.

The beam was simulated using RIAPARMTEQ through the LEBT. The phase space at the entrance of the RFQ is shown in Figure 3. At this location, the beam was bunched and matched into the RFQ acceptance. No transverse emittance growth was observed in the LEBT.
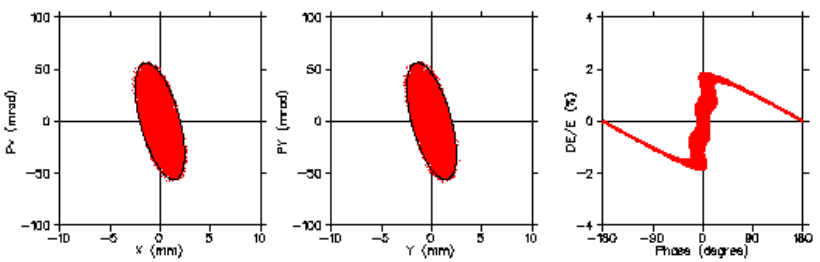

Figure 3: Horizontal (left), vertical (middle), and longitudinal (right) phase space at the entrance of the RFQ.

\section{RFQ}

Benefiting from the higher $\mathrm{Q} / \mathrm{A}$ ratios of the RIBs from the EBIT, the RFQ can achieve an output energy of $\sim 600 \mathrm{keV} / \mathrm{u}$. This energy is higher than the $300 \mathrm{keV} / \mathrm{u}$ used in the driver linac and improves the beam dynamics performance of the superconducting linac due to the increase in longitudinal acceptance. The detailed RFQ design will be discussed in a separate paper [11]. The output phase space from the RFQ is shown in Figure 4. The growth of the transverse emittance in the RFQ is very small whereas tails $(\sim 10 \%$ of the beam) remain in the longitudinal phase spaces after the RFQ. The initial beam energy spread from the EBIT has a significant impact on the $90 \%$ output longitudinal emittance. To meet the requirements of the bunch width and energy spread on target, a small energy spread for the beam from the EBIT is crucial. With an initial beam energy spread of $\pm 0.2 \%$, the $99.5 \%$ longitudinal emittance is within $\sim 2.1 \pi \mathrm{keV} / \mathrm{u}-$ ns. The $90 \%$ emittance is only $0.29 \pi \mathrm{keV} / \mathrm{u}-\mathrm{ns}$ and is comparable to the required longitudinal emittance of 0.25 $\pi \mathrm{keV} / \mathrm{u}-\mathrm{ns}$. The transmission efficiency of the RFQ is about $82 \%$.
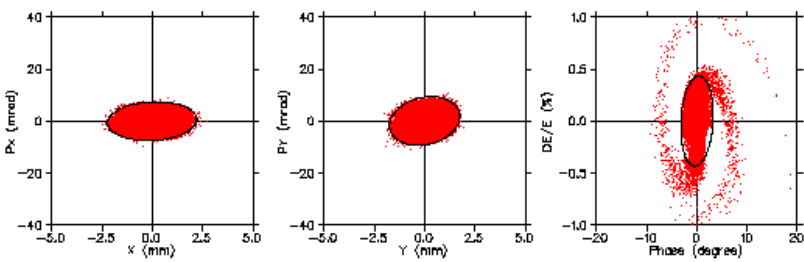

Figure 4: Horizontal (left), vertical (middle), and longitudinal (right) phase space at the exit of the RFQ.

\section{SUPERCONDUCTING LINAC}

A total of three cryomodules consisting of fifteen 80.5 MHz $\lambda / 4$ SRF cavities will be used in the superconducting linac section. Eight superconducting solenoid magnets inside cryomodules will provide the transverse focusing. Each solenoid will have two dipole coils to provide alignment error corrections.

The $1^{\text {st }}$ cryomodule will have two solenoids and one $\lambda / 4$ SRF cavity with a $\beta_{\text {opt }}$ of 0.041 to provide transverse and longitudinal matching into the two following accelerating croyomodules. The $2^{\text {nd }}$ cryomodule will have three solenoids and six $\lambda / 4$ SRF cavities with a $\beta_{\text {opt }}$ of 0.041 to accelerate the RIBs to $\sim 1.2 \mathrm{MeV} / \mathrm{u}$. Since the experimental program may require a RIB energy less than the RFQ output of $600 \mathrm{keV} / \mathrm{u}$, this cryomodule can also be used to decelerate the RIBs to $\sim 300 \mathrm{keV} / \mathrm{u}$. The third cryomodule will have three solenoids and eight $\lambda / 4 \mathrm{SRF}$ cavities with a $\beta_{\text {opt }}$ of 0.085 to accelerate the RIBs up to $\sim 3 \mathrm{MeV} / \mathrm{u}$. for cases with final beam energy below $3 \mathrm{MeV} / \mathrm{u}$, some SRF cavities can be used as rebunchers.

The beam envelope and longitudinal $\beta$ function along the superconducting linac for the case of acceleration to $\sim 3.0 \mathrm{MeV} / \mathrm{u}$ are shown in Figure 5. The linac has adequate transverse and longitudinal acceptance. No transverse emittance growth was observed. The $90 \%$ longitudinal emittance growth was only $\sim 7 \%$. No parametric resonance was observed in the beam simulations. Figure 6 shows the resultant phase space at the exit of the linac.
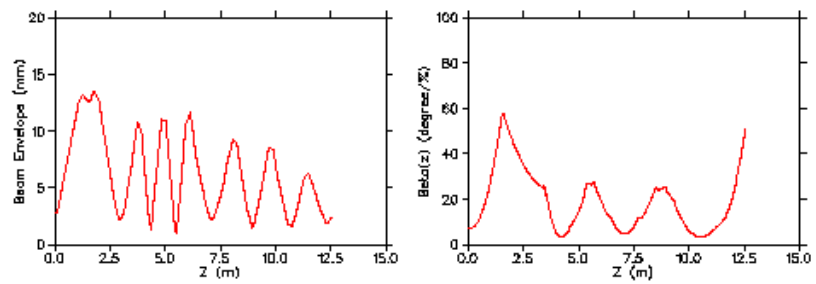

Figure 5: Beam envelope (left) and longitudinal beta function in the linac for RIBs accelerated to $\sim 3.0 \mathrm{MeV} / \mathrm{u}$.

Beam simulations for the case of RIB decelerated to $\sim 300 \mathrm{keV} / \mathrm{u}$ were also performed with similar results. Due to the decrease in beam energy, the longitudinal phase advance is larger but no transverse emittance growth was observed, and the $90 \%$ longitudinal emittance growth was only $\sim 10 \%$. 

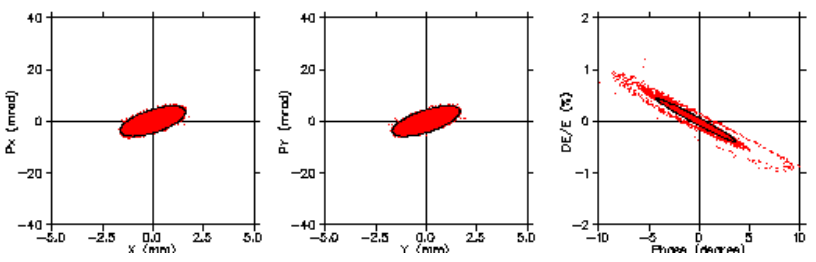

Figure 6: Horizontal (left), vertical (middle), and longitudinal (right) phase space at the exit of linac for RIBs accelerated to $\sim 3.0 \mathrm{MeV} / \mathrm{u}$.

\section{HEBT}

The output beam longitudinal phase space shown in Figure 6 has a very small phase width of about $\pm 5^{\circ}(\sim 0.3$ ns) and a large energy spread of about $\pm 1.0 \%$ with beam energy of about $3.0 \mathrm{MeV} / \mathrm{u}$. To satisfy the requirements of the bunch width and energy spread on target, the HEBT first uses a $\pi$-phase advance cell consisting of four quadrupoles and a cryomodule with a single $\lambda / 4$ SRF cavity with a $\beta_{\text {opt }}$ of 0.041 . The beam bunch will first pass through the $\pi$ phase advance cell and drift longitudinally with adequate transverse focusing. Then the $\lambda / 4$ SRF cavity will rotate the longitudinal phase space, minimizing the beam energy spread. The last segment of the HEBT will be a final focusing cell consisting of four quadrupoles and a superconducting solenoid magnet to achieve a beam spot of $\sim 1 \mathrm{~mm}$ on target. In this cell, the change in bunch length is negligible due to the small energy spread after the rebuncher cavity.

Figure 7 shows the beam phase space and Figure 8 the bunch width and energy spread distributions on the target for a beam accelerated to $3.0 \mathrm{MeV} / \mathrm{u}$. About $88 \%$ of beam on target was within the required bunch width and energy spread of $1 \mathrm{~ns}$ and $1 \mathrm{keV} / \mathrm{u}$ respectively.
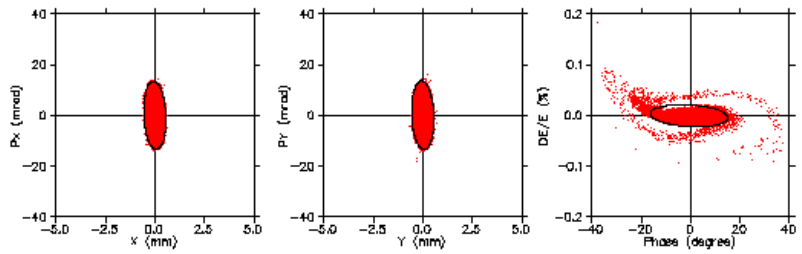

Figure 7: Horizontal (left), vertical (middle), and longitudinal (right) phase space on the target for RIBs accelerated to $3.0 \mathrm{MeV} / \mathrm{u}$.
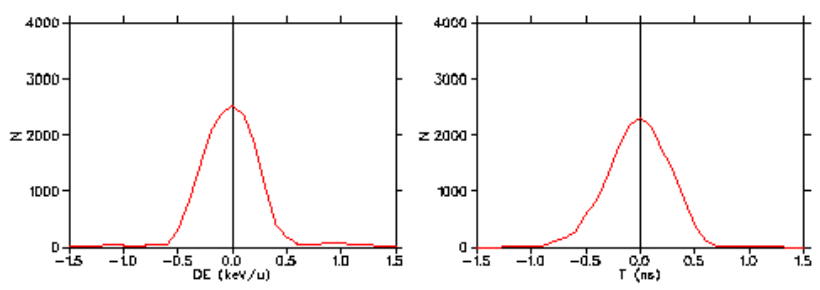

Figure 8: The energy spread (left) and bunch width (right) distributions on the target for beam accelerated to 3.0 $\mathrm{MeV} / \mathrm{u}$.
Beam simulations showed that the performance for the RIBs decelerated to $\sim 300 \mathrm{keV} / \mathrm{u}$ was similar with about $89 \%$ of beam on target within the required bunch width and energy spread. Other cases within the required energy range of $300 \mathrm{keV} / \mathrm{u}$ to $3 \mathrm{MeV} / \mathrm{u}$ were simulated with similar results showing that the accelerator system design meets the performance requirements in all cases.

\section{SUMMARY AND CONCLUSIONS}

End-to-end beam simulations were performed and results show the performance of the proposed NSCL reaccelerator meets the anticipated requirements for the nuclear astrophysics experimental program. An upgrade for beam energies up $\sim 12 \mathrm{MeV} / \mathrm{u}$ is possible by adding additional accelerating cryomodules.

\section{REFERENCES}

[1] G. Bollen, D.J. Morrissey and S. Schwarz, “ A study of a gas-stopping of intense energetic rare isotope beam", Nucl. Instrum. Meth. A 550 (2005) 27.

[2] S. Schwarz, " A high-current EBIT as a charge breeder for the re-acceleration of rare isotopes at the NCSL", to be published at ICIS 2007, Jeju, Korea.

[3] F. Ames et al, "REXEBIS Operation and Developments," Rev. Sci. Instr. 75 (2004) 1607.

[4] O. Kester, private communications.

[5] T. P. Wangler et al. "RIAPAMTQ/IMPACT: BeamDynamics Simulation Tool for RIA," Proceedings of the XXIII Linac Conference, Knoxville, Tennessee, USA, August 2006.

[6] M. Doleans et al. "Improvement of the Longitudinal Beam Dynamics Tuning Procedure for the RIA Driver Linac," Proceedings of 2005 Particle Accelerator Conference, Knoxville, Tennessee, USA, May 2005.

[7] R. Servranckx, K. Brown, L.Schachinger, D. Douglas, "User's Guide to the Program Dimad," SLAC Report 285, UC-28, May 1985.

[8] K. R. Crandall and D. P. Rusthoi, "TRACE 3-D Documentation," LA-UR-97-886, Los Alamos National Laboratory Report, May 1997.

[9] "Isotope Science Facility at Michigan State University - Upgrade of the NSCL rare isotope research capabilities," MSUCL-1345, November, 2006.

[10] W. Hartung et al, "Niobium Quarter-wave Resonator Development for the Rare Isotope Accelerator," Proceedings of the $11^{\text {th }}$ Workshop on the RF Superconductivity, Travemunde, Germany (2003).

[11] Q. Zhao et al, "Design Studies of the Reaccelerator RFQ at the NSCL," these proceedings. 